\title{
Orbital elements of the material surrounding comet 67P/Churyumov-Gerasimenko
}

\author{
B. J. R. Davidsson ${ }^{1}$, P. J. Gutiérrez ${ }^{2}$, H. Sierks ${ }^{3}$, C. Barbieri ${ }^{4}$, P. L. Lamy ${ }^{5}$, R. Rodrigo ${ }^{6,7}$, D. Koschny ${ }^{8}$, H. Rickman ${ }^{1,9}$, \\ H. U. Keller ${ }^{10}$, J. Agarwal ${ }^{3}$, M. F. A'Hearn ${ }^{11,12}$, M. A. Barucci ${ }^{13}$, J.-L. Bertaux ${ }^{14}$, I. Bertini ${ }^{15}$, D. Bodewits ${ }^{11}$, \\ G. Cremonese ${ }^{16}$, V. Da Deppo ${ }^{17}$, S. Debei ${ }^{18}$, M. De Cecco ${ }^{19}$, S. Fornasier ${ }^{13}$, M. Fulle ${ }^{20}$, O. Groussin ${ }^{21}$, C. Güttler ${ }^{3}$, \\ S. F. Hviid ${ }^{22}$, W.-H. Ip ${ }^{23}$, L. Jorda ${ }^{5}$, J. Knollenberg ${ }^{22}$, G. Kovacs ${ }^{3}$, J.-R. Kramm³ , E. Kührt ${ }^{22}$, M. Küppers² \\ F. La Forgia ${ }^{4}$, L. M. Lara ${ }^{2}$, M. Lazzarin ${ }^{4}$, J. J. Lopez Moreno ${ }^{2}$, S. Lowry ${ }^{25}$, S. Magrin ${ }^{26}$, F. Marzari ${ }^{26}$, H. Michalik ${ }^{27}$, \\ R. Moissl-Fraund ${ }^{24}$, G. Naletto ${ }^{28}$, N. Oklay ${ }^{3}$, M. Pajola ${ }^{15}$, C. Snodgrass ${ }^{29}$, N. Thomas ${ }^{30}$, \\ C. Tubiana ${ }^{3}$, and J.-B. Vincent ${ }^{3}$ \\ (Affiliations can be found after the references)
}

Received 7 February 2015 / Accepted 9 April 2015

\begin{abstract}
Context. We investigate the dust coma within the Hill sphere of comet 67P/Churyumov-Gerasimenko.

Aims. We aim to determine osculating orbital elements for individual distinguishable but unresolved slow-moving grains in the vicinity of the nucleus. In addition, we perform photometry and constrain grain sizes.

Methods. We performed astrometry and photometry using images acquired by the OSIRIS Wide Angle Camera on the European Space Agency spacecraft Rosetta. Based on these measurements, we employed standard orbit determination and orbit improvement techniques.

Results. Orbital elements and effective diameters of four grains were constrained, but we were unable to uniquely determine them. Two of the grains have light curves that indicate grain rotation.

Conclusions. The four grains have diameters nominally in the range $0.14-0.50 \mathrm{~m}$. For three of the grains, we found elliptic orbits, which is consistent with a cloud of bound particles around the nucleus. However, hyperbolic escape trajectories cannot be excluded for any of the grains, and for one grain this is the only known option. One grain may have originated from the surface shortly before observation. These results have possible implications for the understanding of the dispersal of the cloud of bound debris around comet nuclei, as well as for understanding the ejection of large grains far from the Sun.
\end{abstract}

Key words. astrometry - celestial mechanics - comets: individual: 67P/Churyumov-Gerasimenko

\section{Introduction}

Three decades ago, observations with radar (Goldstein et al. 1984; Campbell et al. 1989), the IRAS satellite (Sykes et al. 1986), and the Giotto spacecraft (Richter et al. 1991) established that comet nuclei eject macroscopic ( $\mathrm{mm}$ to $\mathrm{dm}$ ) chunks of material into space, henceforth referred to as grains regardless of size, in accordance with the nomenclature used by Rotundi et al. (2015). This inspired several theoretical studies that investigated the existence of gravitationally bound grains that orbit comet nuclei (e.g., Richter \& Keller 1995; Fulle 1997; Scheeres \& Marzari 2000; Crifo et al. 2005; Molina et al. 2008). These studies suggest that grains can remain bound during timescales approaching or exceeding a comet orbital period. Recently, the EPOXI mission to comet 103P/Hartley 2 (hereafter 103P) and the Rosetta mission to comet 67P/Churyumov-Gerasimenko (hereafter 67P) have provided images showing grains as individual point sources. Their observed motions suggest that some of the grains are bound. Specifically, 103P is surrounded by thousands of grains with maximum diameters in the range $0.4 \leq d \leq$ $4 \mathrm{~m}$, and out of 50 well-studied grains located within $15 \mathrm{~km}$ of the nucleus, $10 \%-20 \%$ moved slower than the local escape speed $v_{\text {esc }}$ (A'Hearn et al. 2011; Kelley et al. 2013; Hermalyn et al. 2013). Similarly, five OSIRIS Narrow Angle Camera images acquired on August 4, 2014 at 3.6 AU from the Sun and $275 \mathrm{~km}$ from comet 67P show 48 fast-moving and 350 slow-moving individual grains (Rotundi et al. 2015). Grains in the latter group have an isotropic sub- $v_{\text {esc }}$ velocity distribution once the projected spacecraft velocity is subtracted and strictly radial motion is assumed. This was interpreted by Rotundi et al. (2015) as a cloud of bound grains within ten nucleus radii from 67P. It is also well known that large grains may escape the nucleus, either immediately during the perihelion passage, or later as the cloud of bound grains disperses. Such grains are observed as trails, for instance, for comet 67P (Sykes \& Walker 1992).

It has not been possible before to determine orbital elements of individual comet grains. However, eccentricity determination is required to unambiguously show that a grain is bound. Information about actual grain dynamics is a necessary starting point for orbit integration backward and forward in time. This integration is needed to understand at what time grains were ejected, how their orbits evolved to their current states, and why they eventually escape or impact the nucleus. In the current work we perform astrometry, orbit determination, and orbit improvement to characterize the orbital elements of a handful of grains observed during the Rosetta mission to comet $67 \mathrm{P}$.

\section{Observations}

We here summarize the observations, data reduction, grain identification procedure, and astrometric measurements. The 
OSIRIS Wide Angle Camera (WAC) has a $11.35^{\circ} \times 12.11^{\circ}$ field of view and a 21" pixel resolution (Keller et al. 2007). We used 30 WAC images in the VIS610 filter from the STP017_GRAINS1 sequence, acquired on September 10, 2014 when Rosetta was $28 \mathrm{~km}$ from the comet and at $3.39 \mathrm{AU}$ from the Sun. Image exposures were initiated every $60 \mathrm{~s}$, the first at 04:00:20.854 UTC, the last at 04:29:20.807 UTC, with exposure times of $10.2 \mathrm{~s}$. Exposure time interval midpoints were used for astrometric purposes. The OSIRIS calibration pipeline (Tubiana et al. 2015) was applied; this includes bias subtraction, dark current and bad pixel removal, flat-field division, absolute calibration using standard stars, and correction for geometric distortion. Only such so-called level 3 images were used.

A software package ${ }^{1}$ located point sources in each image, requiring that the signal of the central pixel is at least twice as strong as the median signal in a quadratic frame located between $7-13$ pixels from the central pixel. Storage of CCD $\{x, y\}$ positions of the brightest pixel was only made after visual inspection, excluding obvious cases of cosmic hits, hot pixels, and on-nucleus pixels. Typically, 280-380 point sources were stored for each image. The three manually identified stars $\kappa$ Velorum, $N$ Velorum, and $\iota$ Carinae were used to register all images to a common $\operatorname{CCD}\{x, y\}$-coordinate system. Co-registration required only translation, but no rotation. We classified as stars objects that were registered at the same location to within 3 pixels in at least two images. The final discrepancy in all stellar positions in the images was 0.8 WAC pixels (px) on average, with $70-90 \%$ of the stars located to within 1.5 px and $97-100 \%$ located to within $2.5 \mathrm{px}$ of their nominal positions, with some variation among images as indicated by the ranges.

Objects moving with respect to stars were identified manually by plotting positions from five images at a time. We detected a total of four slow-moving point-like grains, labeled A-D. Figure 1 shows grain $\mathrm{B}$ moving against the stellar background in excerpts of four WAC images. Grains B and C were seen in all 30 images, while grains A and D were seen in 29 images. All measured grain positions, with respect to the co-registered stellar background in CCD $\{x, y\}$-coordinates, are shown in Fig. 2.

Point spread functions (PSFs) shaped as 2D Gaussians were fitted to each grain image by minimizing residuals between observations and fitting functions. The widths in the $x$ and $y$-directions were fitted separately. The FWHM was determined based on the greater of the two widths. We defined $f=F W H M / 2$ as the grain position uncertainty. This is an arbitrary but reasonable assumption, since the grain center of mass probably is located within the region of the relatively strong signal. Minimum, maximum, and median values per grain are denoted by $f_{\min }, f_{\max }$, and $\langle f\rangle$, respectively. The results are summarized in Table 1. The fact that the PSFs are so narrow shows that the grains are not defocused (i.e., they are relatively distant) and they are not smeared measurably by grain motion during the exposures. Since the actual location of grain centers (and stars) within their PSFs are uncertain by up to $1.8 \mathrm{px}$, it is not surprising that registration is sometimes only good to within $\sim 3 \mathrm{px}$.

Conversion from CCD $\{x, y\}$ coordinates to the equatorial system right ascension and declination $\{\alpha, \delta\}$ was made using formulae by Turner (1893), also see Smart (1977). Independent verification tests of the astrometry were made by using the commercial software Astrometrica by Raab (2012) for three images. The positions of grain A agreed to within 1.4, 1.6, and $1.7 \mathrm{px}$ in these test for the three images, which means that our results are

\footnotetext{
If not stated otherwise, all software used for this work was written by B. J. R. Davidsson.
}
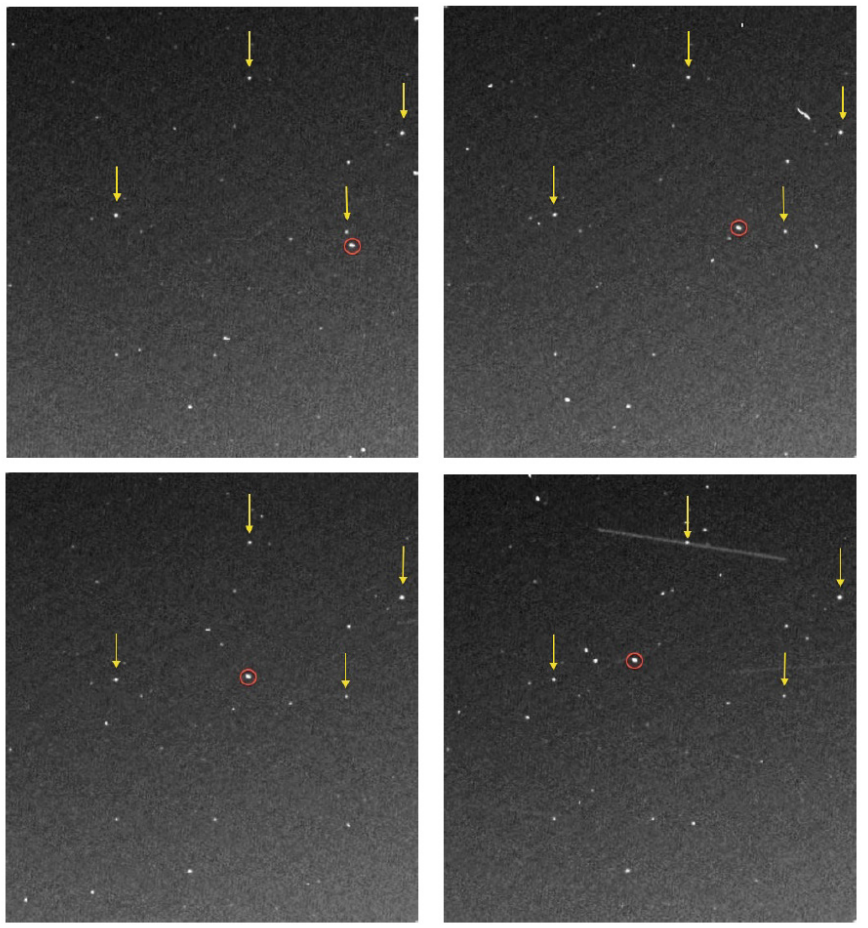

Fig. 1. Grain B (marked with a ring) is moving against the stellar background (four stars marked with arrows). The images were taken $120 \mathrm{~s}$ apart, and these excerpts cover $1.9^{\circ} \times 2.1^{\circ}$. The images contain many fast grains that are smeared to tracks during the exposure (examples in the lower right panel). The images also contain numerous point sources that are not stars, and if they are real, they cannot easily be identified and tracked as individual moving grains in a sequence of images. Original images: WAC_2014-09-10T03.59.12.757Z_ID30_1397549800_F18.fts, WAC_2014-09-10T04.01.12.755Z_ID30_1397549200_F18.fts, WAC_ 2014-09-10T04.03.12.733Z_ID30_1397549400_F18.fts, WAC_201409-10T04.05.12.759Z_ID30_1397549800_F18.fts.

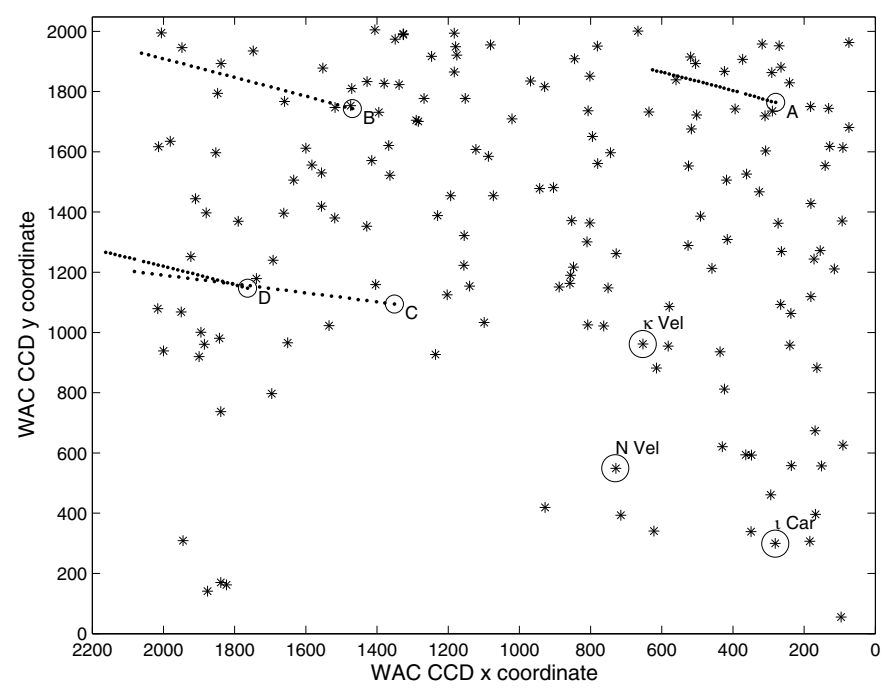

Fig. 2. Objects registered as stars are marked as black asterisks, with $\kappa$ Velorum, $N$ Velorum, and $\iota$ Carinae highlighted individually. Known positions of grains A-D are shown as dots, with the beginning of each track marked by a ring and grain label. The comet nucleus fills a substantial fraction of the original images, here seen as a star-free field.

consistent with a maximum position uncertainty of $\sim 3 \mathrm{px}$ based on registration and PSFs. 
Table 1. PSF-based grain position uncertainty.

\begin{tabular}{lccc}
\hline \hline Grain & $f_{\min }[\mathrm{px}]$ & $f_{\max }[\mathrm{px}]$ & $\langle f\rangle[\mathrm{px}]$ \\
\hline A & 0.80 & 1.59 & 1.05 \\
B & 1.02 & 1.53 & 1.21 \\
C & 1.06 & 1.80 & 1.46 \\
D & 0.75 & 1.52 & 1.04 \\
\hline
\end{tabular}

\section{Methods}

We here summarize the pipeline used to obtain orbital elements and describe the photometric measurements. The pipeline consists of preliminary orbit determination, followed by two levels of orbit improvement. Orbit determination was made using the Gauss method as described by Danby (1989). As input, the Gauss method uses three astrometric positions and the position vectors of the observer at the time of observations, seen from the center of gravity (here, the comet). The latter vectors were obtained from SPICE kernels provided by ESA/ESOC and prepared by P. J. Gutiérrez. A variant of the Gaussian gravitational constant was used, evaluated for a comet mass of $M_{\text {nuc }}=$ $1.0 \times 10^{13} \mathrm{~kg}$ (Sierks et al. 2015), so that lengths are measured in kilometers, time in hours, and mass in units of $M_{\text {nuc }}$. The output, after an iterative procedure, is three cometocentric position vectors of the observed target that can be used to determine an approximate target velocity vector for the second observation, which is sufficient to calculate orbital elements.

Unfortunately, the Gauss method does not converge to a physical solution for any considered position triplet for any grain. This is a common problem when the arc length is too short or the positions lie too close to a great circle (Marsden 1985). A standard approach to facilitate convergence is to replace the automatically generated initial guess of the observertarget distance $\Delta_{2}^{0}$ at the second observation by a manually provided value that is closer to the real one. The pipeline considers the $1 \leq \Delta_{2}^{0} \leq 100 \mathrm{~km}$ region with $1 \mathrm{~m}$ spatial resolution and counts the number of iterations $k$ for which the observer-target distance $\Delta_{2}^{k}$ remains to within $0.5 \mathrm{~km}$ of the initial guess.

Figure 3 shows the result for grain B. For most initial values the divergence is immediate, but in two regions at $17.3 \leq$ $\Delta_{2}^{0} \leq 20.3 \mathrm{~km}$ and $34.8 \leq \Delta_{2}^{0} \leq 37.1 \mathrm{~km}$, roughly symmetrically placed around the distance to the nucleus, quasi-stable conditions prevail before divergence. This indicates that the calculated grain position vectors almost fulfill the two physical constraints placed on the geometry by the Gauss method (that the vectors are co-planar and that they obey Kepler's second law).

The pipeline calculates preliminary orbital elements for each initial guess that has resulted in quasi-stable conditions (defined as $k \geq 2$ ). Grain position vectors of the last stable iteration are used as a starting point.

Orbit improvement was made using the pseudo-Newton variant of the differential correction algorithm (Danby 1989; Sansaturio et al. 1996). In that algorithm, small adjustments of the preliminary orbital elements are made with the goal of minimizing residuals between astrometry and ephemerides calculated from the improved orbital elements. Orbit improvement was made in two steps. First, the pipeline considers the current triplet. On average, the astrometry can be reproduced to within $0.01-0.1$ WAC pixels. This confirms that the divergence of the Gauss method does not occur because there are no orbital element solutions. Second, the orbit improvement is applied using the full set of astrometric positions available for a given grain.

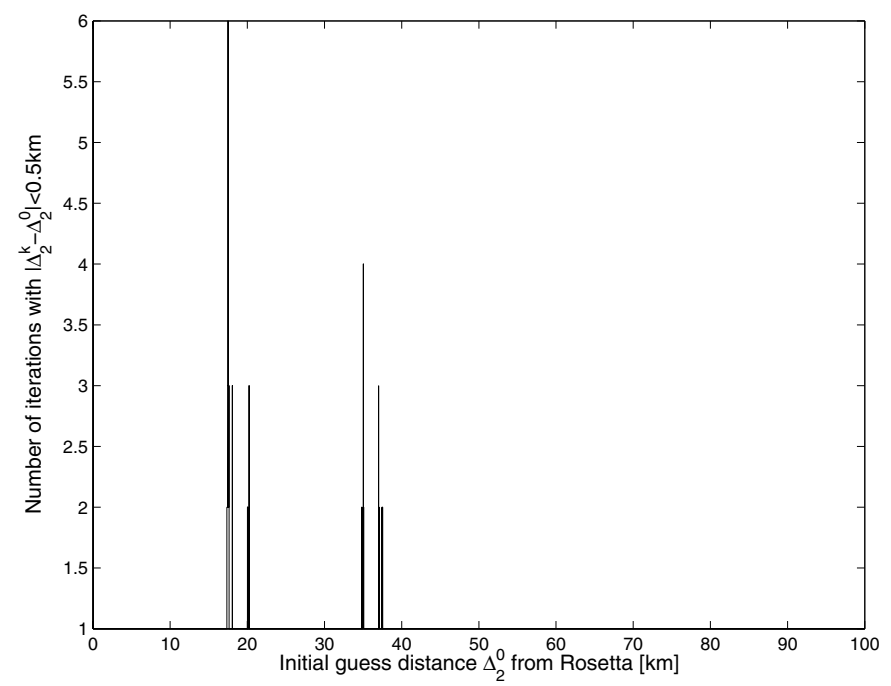

Fig. 3. Number of Gauss method iterations before divergence as a function of initial guess $\Delta_{2}^{0}$ for grain B. Quasi-converging behavior is seen in two regions, roughly symmetrically placed around the spacecraftnucleus distance of $28 \mathrm{~km}$. This provides sufficient information to determine preliminary orbital elements that subsequently are refined using orbit improvement techniques.

The pipeline was run for several different triplets per grain to search the parameter space as well as possible. We obtained numerous solutions, all with average residuals of $\lesssim 1 \mathrm{px}$ and local maximum residuals of $\$ 3 \mathrm{px}$. We consider all these solutions to be consistent with the astrometry, given the position uncertainties.

The photometry of each grain was made as follows. A circular aperture with 4 px radius was centered on the grain. The mean radiance within the previously mentioned quadratic frame was subtracted from the pixels within the circular aperture. The sum of remaining radiance within the circular aperture was taken as the radiance of the grain. The background is dominated by illuminated unresolved dust, and clearly distinguishable jets from the nucleus stretch across the images. Subtraction of the background level risks inadvertent removal of real signal from the grains, since the dust column between OSIRIS and the grain may be shorter than that of the nearby background. For this reason, our grain sizes are lower limits. When calculating the grain sizes, we applied a solar spectral irradiance of $1.709 \mathrm{~W} \mathrm{~m}^{-2} \mathrm{~nm}^{-1}$ at the filter central wavelength $\lambda=612.7 \mathrm{~nm}$ (Colina et al. 1996), an albedo $A=0.059$ (Sierks et al. 2015) identical to that of the nucleus, a WAC image scale of $101 \mu \mathrm{rad} \mathrm{px}^{-1}$ (Keller et al. 2007), and we assumed spherical grains with a phase function according to Eq. (A5) in Bowell et al. (1989). Grain distances and phase angles follow from our orbit solutions.

\section{Results}

We here discuss the results, primarily using grain B as an illustrative example, and present orbital elements, sizes, and related information for all grains in tabulated form.

For grain $\mathrm{B}$ we obtained 800 solutions called $\mathrm{B}_{1}$ for $17.3 \leq$ $\Delta_{2}^{0} \leq 20.3 \mathrm{~km}$ (i.e., between Rosetta and the nucleus) with semimajor axes $-10.1 \leq a \leq-1.9 \mathrm{~km}$, and 434 solutions called $\mathrm{B}_{2}$ for $34.8 \leq \Delta_{2}^{0} \leq 37.1 \mathrm{~km}$ (i.e., beyond the nucleus) with semimajor axes $-0.65 \leq a \leq-0.36 \mathrm{~km}$. Figure 4 shows the mean and maximum residuals, $\langle\rho\rangle$ and $\rho_{\max }$, for these sets of orbital 


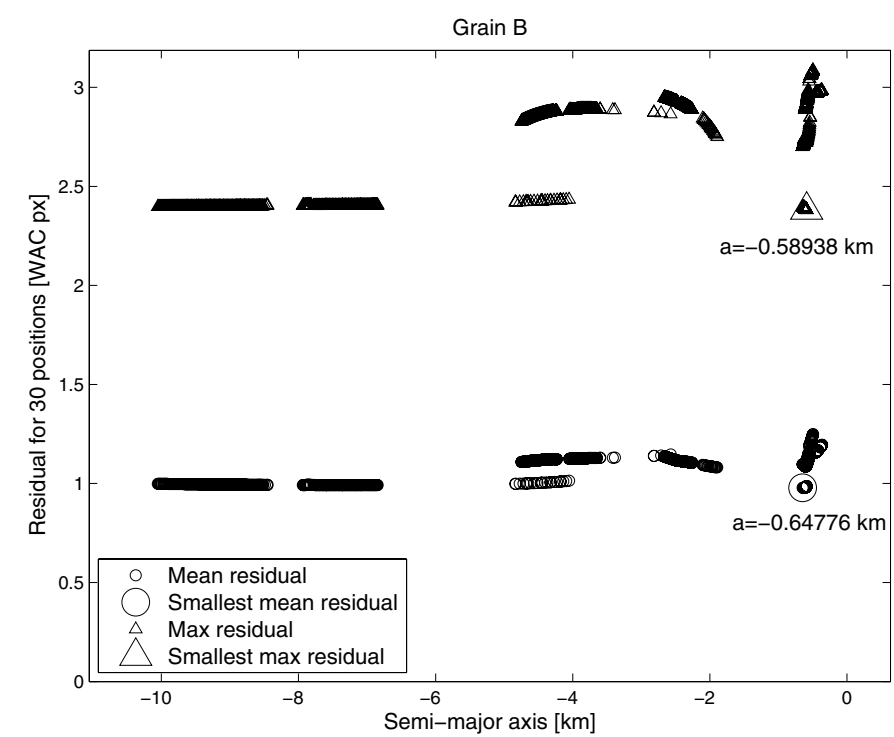

Fig. 4. Maximum and mean residuals for grain B versus semi-major axis $a$. The smallest maximum and mean residuals are marked, along with the corresponding $a$ value.

elements, plotted versus $a$. It is clear that 1) all solutions consistently place grain B on a hyperbolic escape trajectory from the nucleus and that 2) all these solutions must be considered equally plausible since residuals are within the position uncertainties, and there is no significant difference between the orbital elements with the smallest residuals and the rest. Thus, no preference for the two groups of solutions can be stated purely based on residuals. However, we note that cluster $\mathrm{B}_{1}$ is twice as large as cluster $\mathrm{B}_{2}$, and on average, the initial distances of $\mathrm{B}_{1}$ cause more Gauss method iterations before divergence than those of $\mathrm{B}_{2}$.

According to Fig. 2, grain B has the second fastest apparent motion of the grains. If grain $B$ is relatively close to Rosetta $\left(B_{1}\right)$, then a larger part of the apparent motion is due to parallax (caused by Rosetta's movement) and a smaller fraction is due to grain orbital velocity. However, if the grain is farther away $\left(\mathrm{B}_{2}\right)$, the parallax becomes less important, and a larger part of the apparent motion must be due to a relatively high orbital grain velocity. For hyperbolic orbits, the orbital velocity (at a given distance from the nucleus) decreases when $|a|$ increases, according to the vis-viva law (Danby 1989). That is why the $|a|$ values are higher for $\mathrm{B}_{1}$ than for $\mathrm{B}_{2}$, and it is clear that a relatively high apparent velocity of a grain does not necessarily mean that it is close.

Figure 5 shows the eccentricity $e$ and the quantity $T_{0}-T_{\text {per }}$, where $T_{0}$ is an epoch time (taken as JD 2456910.6667 and corresponding to September 10, 2014, 04:00:00 UTC) and $T_{\text {per }}$ is the pericenter passage time of the grain. The $\mathrm{B}_{1}$ eccentricities are relatively low and well constrained $(e=3.34 \pm 1.25)$, while the $\mathrm{B}_{2}$ eccentricities are substantially higher and more diverse $(e=12.92 \pm 6.32)$. A high degree of orbit curvature is needed to explain the observed trajectory, and more so for $\mathrm{B}_{2}$ than for $\mathrm{B}_{1}$. Grain B has a significant angular momentum with respect to the nucleus and does not have a purely radial motion.

It may be surprising that for some orbit solutions, $T_{0}-T_{\text {per }}<0$, which means that the grain on a hyperbolic orbit is approaching its pericenter. However, these pre-pericenter solutions are not necessarily correct. There is a fairly large uncertainty in $T_{\text {per }}$ because that parameter is not entirely independent of the argument of pericenter $\omega$, plotted in Fig. 6. Since $\omega$ measures the angular distance from the ascending node vector to the
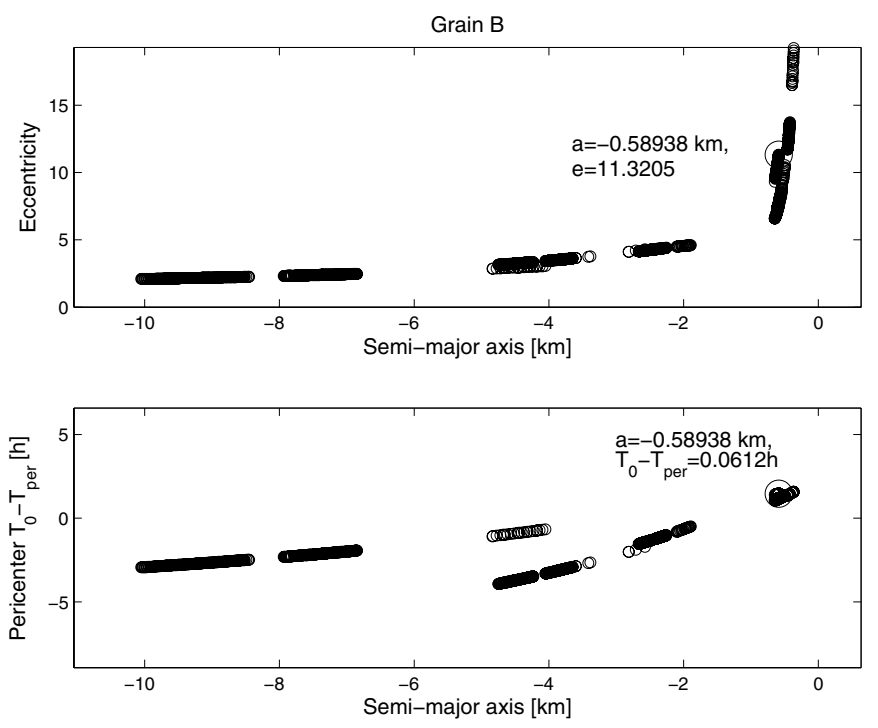

Fig. 5. Upper panel: eccentricity $e$ of grain B versus semi-major axis $a$. Lower panel: the time $T_{0}-T_{\text {per }}$ that has passed since the pericenter crossing $T_{\text {per }}$ until the epoch $T_{0}=2456910.6667 \mathrm{JD}$, versus $a$. The values of $e$ and $T_{0}-T_{\text {per }}$ corresponding to the lowest value of the maximum residual are highlighted.
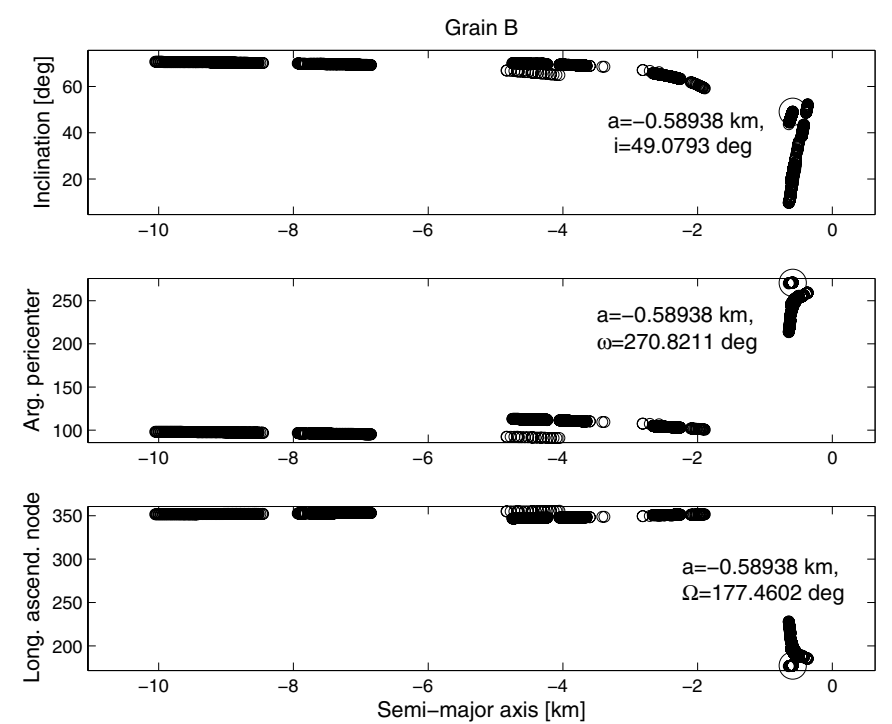

Fig. 6. Upper panel: inclination $i$ versus semi-major axis a. Middle panel: argument of pericenter $\omega$ versus $a$. Lower panel: longitude of the ascending node $\Omega$ versus $a$. The values corresponding to the smallest local maximum residual are highlighted.

pericenter, while $T_{0}-T_{\text {per }}$ corresponds to a certain true anomaly $v$ (angular distance from the pericenter to the grain at the epoch), it is possible that widely differing combinations of $\omega$ and $T_{\text {per }}$ correspond to rather similar grain positions in space at a particular epoch. To test this hypothesis, we calculated the argument of the latitude $u=v+\omega$ and found that it has a spread of $3.2^{\circ}$ for $\mathrm{B}_{1}$. This is smaller than the spread in $\omega$-values, $11.2^{\circ}$. The argument of the latitude is more tightly constrained than $\omega$ and $T_{\text {per }}$ individually. For a short orbital arc, it is difficult to simultaneously and reliably constrain the location of the pericenter point and the time of the pericenter passage. Thus, low negative $T_{0}-T_{\text {per }}$ values are potentially unphysical and related to corresponding overestimates of $\omega$. A well-constrained $u$, when $\omega$ and $T_{\text {per }}$ vary considerably, is particularly evident in elliptic solutions for some of the other grains. 

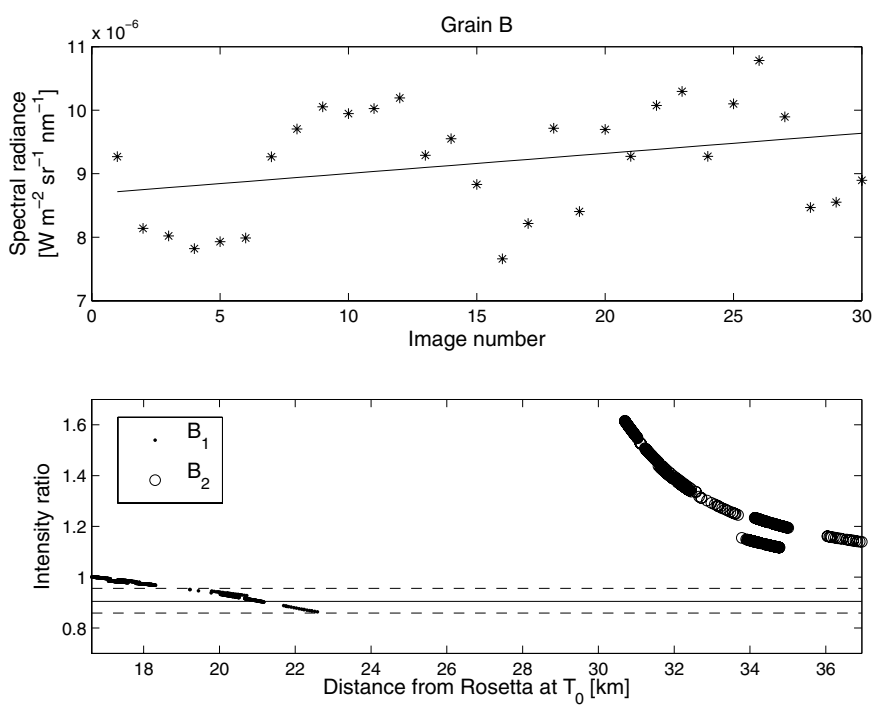

Fig. 7. Upper panel: spectral radiance of grain B versus image number. The line is a least-squares fit, which indicates that grain B brightens with time due to decreasing distance and/or increasing cross section. Lower panel: the parameter $\Phi / r_{\mathrm{s} / \mathrm{c}}^{2}$ has been evaluated for each $\mathrm{B}_{1}$ and $\mathrm{B}_{2}$ orbit, and the ratio between this parameter at the beginning and end of the track is plotted versus $r_{\mathrm{s} / \mathrm{c}}$ at $T_{0}$.

The orientation of the orbital plane is described by the inclination $i$ and longitude of the ascending node $\Omega$, shown in Fig. 6. They are tightly constrained for $\mathrm{B}_{1}\left(i=64.9^{\circ} \pm 5.7^{\circ}\right.$, $\left.\Omega=351.4^{\circ} \pm 4.3^{\circ}\right)$, but a wider variety of solutions are compatible with the astrometry for $\mathrm{B}_{2}\left(i=31.0^{\circ} \pm 21.3^{\circ}, \Omega=\right.$ $202.5^{\circ} \pm 25.7^{\circ}$ ). We also calculated the angle $\beta$ between the angular momentum vector of the grain orbits and the positive spin pole $\{\alpha, \delta\}=\left\{69.370^{\circ}, 64.132^{\circ}\right\}$ of the comet nucleus (Sierks et al. 2015) (equivalently, $\beta$ is the angle between the comet equatorial plane and the grain orbital plane). This angle is $\beta=116.5^{\circ} \pm 9.3^{\circ}$ for $\mathrm{B}_{1}$, suggesting that the orbital motion of grain $\mathrm{B}$ is opposite to that of nucleus rotation. For $\mathrm{B}_{2}$ we have $\beta=23.0^{\circ} \pm 16.1^{\circ}$; this trajectory is nearly perpendicular to that of $B_{1}$. It is not surprising that the two solutions differ strongly, considering they both need to reproduce the same astrometry, but with very different parallax contributions.

Although the orbital elements have been presented as ranges, this does not mean that any random combination of orbital elements selected from within these ranges will yield small residuals. Tests were made in which a particular set of orbital elements provided a good fit to the astrometry. The orbital elements were then changed, one at a time, to show the effect on the residual. We found that the quality deteriorates very quickly. If any of the angular elements $\{i, \omega, \Omega\}$ changes by just a few degrees, the mean residuals become unacceptably large. If the semi-major axis changes by a few tens of meters, the eccentricity by a few parts of a hundred, or the time of the pericenter passage by a few tens of minutes, the fit is ruined. This shows that very specific combinations of the orbital elements are necessary.

Of the four grains studied here, grain B is the brightest. The upper panel of Fig. 7 shows the measured background-subtracted spectral radiance of grain B versus image number, that is, as a function of time. The peak value is $41 \%$ higher than the lowest value, and the variation is not random, but appears to have a sinusoidal shape. If the reason is cross-section variation due to grain rotation, this would indicate a rotation period of about $26 \mathrm{~min}$. For $\mathrm{B}_{1}$, the average spectral radiance corresponds to a grain diameter of $d=0.28 \pm 0.02 \mathrm{~m}$ where the uncertainty here is only due to the Rosetta-grain distance for the various orbits, that is, $r_{\mathrm{s} / \mathrm{c}}=18.4 \pm 1.8 \mathrm{~km}$ at the time of the first image. If the lowest and highest radiance values are considered together with distance uncertainty, the full range is $0.24 \leq d \leq 0.31 \mathrm{~m}$. For $\mathrm{B}_{2}$, the average radiance yields $d=0.30 \pm 0.10 \mathrm{~m}$, with a larger uncertainty than for $\mathrm{B}_{1}$ because of the less constrained distance $r_{\mathrm{s} / \mathrm{c}}=32.8 \pm 2.1 \mathrm{~km}$. The full range is $0.20 \leq d \leq 0.46 \mathrm{~m}$. The size estimates for $\mathrm{B}_{1}$ and $\mathrm{B}_{2}$ are very similar in spite of the significant difference in distance. This is because $\mathrm{B}_{1}$ orbits yield substantially larger phase angles than $\mathrm{B}_{2}$, which means that a smaller distance is compensated for, since a smaller fraction of the illuminated hemisphere is visible.

Each set of orbital elements predicts specific changes in $r_{\mathrm{s} / \mathrm{c}}$ and the phase function $\Phi$ during the $30 \mathrm{~min}$ of observation. The lower panel in Fig. 7 shows the value of $\Phi / r_{\mathrm{s} / \mathrm{c}}^{2}$ evaluated at the beginning of the observations, divided by the same quantity evaluated at the end of the observations. The $\mathrm{B}_{1}$ orbits predict a weak ( $\$ 14 \%$ ) brightening of grain $\mathrm{B}$, since a reduction of the phase angle is almost fully compensated for by an increase of the distance. The $\mathrm{B}_{2}$ orbits predict that grain $\mathrm{B}$ becomes dimmer by up to $62 \%$ because both distances and phase angles are increasing. The upper panel of Fig. 7 shows a least-squares fit to the spectral radiances, which indicates that grain B brightens by $\sim 10 \%$. This empirical value, along with error margins based on the standard deviation of the fitted slope, is drawn as horizontal lines in the lower panel of Fig. 7. Solution $\mathrm{B}_{1}$ appears to be more consistent with the observations than solution $\mathrm{B}_{2}$. However, caution is required for three reasons: 1) the background-removal procedure may have influenced the slope; 2) if the grain has excited spin, then systematic changes of the grain cross-section may affect the brightness in addition to distance and phase angle; and 3) the applied phase function may not be adequate for these particular grains.

The pericenter distance $q$ of the orbit is readily obtained from $a$ and $e$. We obtained $6.8 \leq q \leq 12.4 \mathrm{~km}$ for $\mathrm{B}_{1}$ and $3.6 \leq q \leq$ $5.8 \mathrm{~km}$ for $\mathrm{B}_{2}$. The comet-grain distances at $T_{0}$ are $6.9 \leq r_{\text {com }} \leq$ $13.9 \mathrm{~km}$ for $\mathrm{B}_{1}$ and $5.5 \leq r_{\text {com }} \leq 8.9 \mathrm{~km}$ for $\mathrm{B}_{2}$, indicating that grain $\mathrm{B}$ was imaged close to its pericenter. However, the nucleus surface extends at most $2.8 \mathrm{~km}$ from the center. Thus, none of the grain B orbits intercept the nucleus surface. We return to this question in Sect. 5, but first we briefly summarize our results for grains A, C, and D. Information on all our solutions for grain A-D are collected in Tables 2 and 3.

Grain $\mathrm{A}$ is the faintest of the four grains studied here (the spectral radiance is 2.8 times lower than for grain $\mathrm{B}$ ). The light curve is scattered, with no trace of periodicity. Grain A has a diameter of $d \approx 0.15 \mathrm{~m}$. We derived three possible orbital element solutions, with $\mathrm{A}_{1}$ and $\mathrm{A}_{2}$ placing the grain closer to Rosetta than the nucleus, while $A_{3}$ places it beyond the nucleus. $A_{1}$ is a bound polar orbit with modest eccentricity and an orbital period of $28-57 \mathrm{~h} . \mathrm{A}_{2}$ and $\mathrm{A}_{3}$ are hyperbolic orbits, all with $q \geq 4.9 \mathrm{~km}$, which means that they do not intercept the nucleus surface. The light curve indicates a 0-27\% dimming of grain A, which is only consistent with solution $\mathrm{A}_{3}$.

Grain $\mathrm{C}$ is almost as bright as grain $\mathrm{B}$ and shows signs of light-curve periodicity. Three possible orbit solutions were found. $\mathrm{C}_{1}$ yields the shortest grain-Rosetta distance of the entire study, $r_{\mathrm{s} / \mathrm{c}} \approx 11 \mathrm{~km}$, and is elliptic with modest eccentricity. $\mathrm{C}_{2}$ at $r_{\mathrm{s} / \mathrm{c}} \approx 20 \mathrm{~km}$ and $\mathrm{C}_{3}$ at $r_{\mathrm{s} / \mathrm{c}} \approx 37 \mathrm{~km}$ are both hyperbolic, with pericenters that do not intercept the nucleus surface. All dynamic solutions predict constant brightness of grain $\mathrm{C}$ to within $5 \%$ during the $30 \mathrm{~min}$ of observation. However, the observed lightcurve has a $13 \%$ nominal dimming, and even if the standard deviation uncertainty of the slope is considered, this still indicates 
Table 2. Osculating orbital elements and residuals.

\begin{tabular}{|c|c|c|c|c|c|c|c|c|}
\hline Grain & $a[\mathrm{~km}]$ & $e$ & $i\left[^{\circ}\right]$ & $\omega\left[^{\circ}\right]$ & $\Omega\left[{ }^{\circ}\right]$ & $T_{0}-T_{\text {per }}[\mathrm{h}]$ & $\langle\rho\rangle[\mathrm{px}]$ & $\rho_{\max }[\mathrm{px}]$ \\
\hline$\overline{A_{1}}$ & $7.6 \pm 2.0$ & $0.31 \pm 0.15$ & $40.4 \pm 7.8$ & $205.2 \pm 37.5$ & $320.1 \pm 9.8$ & $37.4 \pm 19.5$ & $0.78 \pm 0.06$ & $1.54 \pm 0.22$ \\
\hline $\mathrm{A}_{2}$ & -49.8 to -5.9 & $1.51 \pm 0.40$ & $43.7 \pm 3.5$ & $164.0 \pm 3.2$ & $296.5 \pm 6.0$ & $-2.0 \pm 1.2$ & $0.96 \pm 0.21$ & $2.12 \pm 0.15$ \\
\hline $\mathrm{A}_{3}$ & -294 to -1.6 & $2.59 \pm 1.57$ & $31.7 \pm 10.2$ & $215.4 \pm 35.6$ & $213.0 \pm 37.1$ & $1.84 \pm 0.84$ & $0.87 \pm 0.15$ & $1.88 \pm 0.34$ \\
\hline $\mathrm{B}_{1}$ & -10.1 to -1.9 & $3.34 \pm 1.25$ & $64.9 \pm 5.7$ & $102.0 \pm 11.2$ & $351.4 \pm 4.3$ & $-2.2 \pm 1.7$ & $1.07 \pm 0.08$ & $2.7 \pm 0.3$ \\
\hline $\mathrm{B}_{2}$ & -0.65 to -0.36 & $12.92 \pm 6.32$ & $31.0 \pm 21.3$ & $242.2 \pm 28.6$ & $202.5 \pm 25.7$ & $1.3 \pm 0.3$ & $1.10 \pm 0.12$ & $2.78 \pm 0.40$ \\
\hline $\mathrm{C}_{1}$ & $19.3 \pm 4.8$ & $0.37 \pm 0.05$ & $84.7 \pm 0.5$ & $325.3 \pm 40.2$ & $8.09 \pm 2.0$ & $24.7 \pm 8.7$ & $1.14 \pm 0.02$ & $2.52 \pm 0.03$ \\
\hline $\mathrm{C}_{2}$ & -12.9 to -2.8 & $2.56 \pm 0.66$ & $78.6 \pm 5.8$ & $56.4 \pm 8.7$ & $19.2 \pm 3.7$ & $1.4 \pm 1.2$ & $1.15 \pm 0.09$ & $2.75 \pm 0.26$ \\
\hline $\mathrm{C}_{3}$ & -0.64 to -0.27 & $35.6 \pm 25.8$ & $67.4 \pm 8.0$ & $297.0 \pm 4.3$ & $183.6 \pm 4.5$ & $0.34 \pm 0.17$ & $1.13 \pm 0.08$ & $2.67 \pm 0.18$ \\
\hline$\overline{D_{1}}$ & $5.4 \pm 1.0$ & $0.29 \pm 0.14$ & $65.0 \pm 3.7$ & $173.8 \pm 171.2$ & $354 \pm 2.3$ & $3.3 \pm 1.7$ & $0.86 \pm 0.14$ & $1.95 \pm 0.54$ \\
\hline $\mathrm{D}_{2}$ & $4.1 \pm 0.4$ & $0.50 \pm 0.13$ & $62.2 \pm 4.1$ & $338.5 \pm 13.2$ & $358.6 \pm 3.0$ & $3.1 \pm 1.5$ & $0.80 \pm 0.07$ & $1.59 \pm 0.25$ \\
\hline $\mathrm{D}_{3}$ & 7-1450 & $0.96 \pm 0.04$ & $26.6 \pm 23.7$ & $192.0 \pm 152.6$ & $183.1 \pm 157.3$ & $1.9 \pm 0.3$ & $0.81 \pm 0.08$ & $1.72 \pm 0.36$ \\
\hline $\mathrm{D}_{4}$ & -4750 to -8.9 & $1.07 \pm 0.07$ & $24.7 \pm 21.8$ & $169.4 \pm 52.1$ & $217.6 \pm 46.7$ & $1.84 \pm 0.25$ & $0.92 \pm 0.12$ & $2.09 \pm 0.41$ \\
\hline
\end{tabular}

Notes. Semi-major axis $a$, eccentricity $e$, inclination $i$, argument of pericenter $\omega$, longitude of the ascending node $\Omega$, time elapsed from pericenter passage to epoch $T_{0}-T_{\text {per }}$, mean residual $\langle\rho\rangle$, maximum residual $\rho_{\max }$.

Table 3. Auxiliary grain information.

\begin{tabular}{cccccccccc}
\hline \hline Grain & $\beta\left[^{\circ}\right]$ & $u\left[^{\circ}\right]$ & $q[\mathrm{~km}]$ & $Q[\mathrm{~km}]$ & $P[\mathrm{~h}]$ & $r_{\mathrm{com}}[\mathrm{km}]$ & $r_{\mathrm{s} / \mathrm{c}}[\mathrm{km}]$ & $\alpha_{\mathrm{p}}\left[^{\circ}\right]$ & $d[\mathrm{~m}]$ \\
\hline $\mathrm{A}_{1}$ & $87.3 \pm 9.5$ & $98.1 \pm 10.2$ & $4.7 \pm 1.0$ & $9.9 \pm 2.9$ & $42.6 \pm 14.6$ & $7.2 \pm 1.2$ & $22.7 \pm 1.7$ & $58 \pm 11$ & $0.15_{-0.05}^{+0.04}$ \\
$\mathrm{~A}_{2}$ & $82.7 \pm 6.0$ & $116.3 \pm 8.5$ & $5.5 \pm 0.10$ & - & - & $6.8 \pm 1.3$ & $23.2 \pm 0.6$ & $56 \pm 27$ & $0.14_{-0.11}^{+0.05}$ \\
$\mathrm{~A}_{3}$ & $33.7 \pm 25.1$ & $250.7 \pm 41.7$ & $5.2 \pm 0.3$ & - & - & $6.2 \pm 0.7$ & $31.1 \pm 1.4$ & $35 \pm 6$ & $0.14_{-0.04}^{+0.07}$ \\
\hline $\mathrm{B}_{1}$ & $116.5 \pm 9.3$ & $78.4 \pm 3.2$ & $9.6 \pm 2.8$ & - & - & $10.4 \pm 3.5$ & $18.4 \pm 1.8$ & $73 \pm 15$ & $0.28_{-0.04}^{+0.03}$ \\
$\mathrm{~B}_{2}$ & $23.0 \pm 16.1$ & $303.6 \pm 37.2$ & $4.7 \pm 1.1$ & - & - & $7.2 \pm 1.7$ & $33.1 \pm 2.4$ & $38 \pm 12$ & $0.30_{-0.10}^{+0.16}$ \\
\hline $\mathrm{C}_{1}$ & $129.8 \pm 0.2$ & $64.9 \pm 0.03$ & $11.7 \pm 3.0$ & $24.6 \pm 4.3$ & $167 \pm 49$ & $16.8 \pm 0.8$ & $11.4 \pm 0.8$ & $86 \pm 1$ & $0.22_{-0.02}^{+0.03}$ \\
$\mathrm{C}_{2}$ & $120.6 \pm 7.3$ & $68.6 \pm 2.5$ & $8.2 \pm 2.4$ & - & - & $8.2 \pm 2.3$ & $20.2 \pm 3.9$ & $76 \pm 8$ & $0.32_{-0.05}^{+0.03}$ \\
$\mathrm{C}_{3}$ & $22.4 \pm 8.4$ & $309.2 \pm 5.2$ & $9.1 \pm 3.4$ & - & - & $9.3 \pm 3.4$ & $36.7 \pm 3.6$ & $65 \pm 8$ & $0.50_{-0.13}^{+0.25}$ \\
\hline $\mathrm{D}_{1}$ & $114.1 \pm 4.6$ & $96.3 \pm 12.7$ & $4.0 \pm 1.1$ & $6.9 \pm 0.8$ & $27.0 \pm 7.0$ & $4.7 \pm 1.2$ & $24.5 \pm 0.8$ & $60 \pm 11$ & $0.24_{-0.05}^{+0.06}$ \\
$\mathrm{D}_{2}$ & $111.1 \pm 5.7$ & $104.1 \pm 23.0$ & $2.2 \pm 0.9$ & $6.1 \pm 0.2$ & $18.2 \pm 3.4$ & $4.5 \pm 1.5$ & $25.2 \pm 0.8$ & $54 \pm 9$ & $0.22_{-0.04}^{+0.06}$ \\
$\mathrm{D}_{3}$ & $27.7 \pm 25.5$ & $339.7 \pm 168.7$ & $0.7 \pm 0.2$ & $13.3-678$ & $40-1220$ & $4.0 \pm 0.2$ & $30.0 \pm 0.7$ & $40 \pm 4$ & $0.21_{-0.04}^{+0.06}$ \\
$\mathrm{D}_{4}$ & $29.7 \pm 26.1$ & $324.7 \pm 76.6$ & $0.8 \pm 0.1$ & - & - & $4.3 \pm 0.2$ & $30.4 \pm 0.5$ & $41 \pm 2$ & $0.23_{-0.04}^{+0.07}$ \\
\hline
\end{tabular}

Notes. Angle between comet equatorial and grain orbital planes $\beta$, argument of latitude $u$, pericenter distance $q$, apocenter distance $Q$, orbital period $P$, grain-comet distance $r_{\mathrm{com}}$ at epoch $T_{0}$, grain-Rosetta distance $r_{\mathrm{s} / \mathrm{c}}$ at epoch $T_{0}$, phase angle $\alpha_{\mathrm{p}}$, grain diameter $d$.

a dimming of at least $8 \%$. This discrepancy is most likely due to cross-section changes, background removal, and/or phase function inaccuracy. With increasing distance, the size prediction for grain $\mathrm{C}$ is $d \approx 0.2 \mathrm{~m}, d \approx 0.3 \mathrm{~m}$, or $d \approx 0.5 \mathrm{~m}$.

Grain D is about twice as bright as grain A, and the light curve has no trace of periodicity. In spite of having four very different possible orbital solutions, the size estimates are very similar, $d \approx 0.2 \mathrm{~m}$. Three of the solutions are ellipses. Of these, $\mathrm{D}_{1}$ has a modest eccentricity and a pericenter point located outside the nucleus surface. $\mathrm{D}_{2}$ has a somewhat higher eccentricity, and a sufficiently small pericenter distance to suggest grain impact on the nucleus after one revolution. These two solutions locate grain D on the near-side of the nucleus as seen from Rosetta. There is also a family of elliptic solutions $\mathrm{D}_{3}$ that places grain $\mathrm{D}$ on the far side of the nucleus. They all have eccentricities just below unity and lead to nucleus impact after an excursion to the apocenter distance, which in some cases is located outside the Hill radius that equals $602 \mathrm{~km}$ at the considered heliocentric distance. Finally, $\mathrm{D}_{4}$ is hyperbolic and constitutes the only $e>1$ case in this study for which the pericenter is located within the comet nucleus. The light curve shows that grain $\mathrm{D}$ becomes dimmer by $\sim 15 \%$ during the observation sequence. This behavior is consistent with solutions $\mathrm{D}_{3}$ and $\mathrm{D}_{4}$, but not with the two solutions that place grain $\mathrm{D}$ relatively close to Rosetta.

\section{Discussion}

The Gauss method as well as the orbit improvement technique used here assume that particles move solely under the influence of nucleus gravity originating from a point source, meaning that the potential field is $\propto r_{\text {com }}^{-1}$. In reality, the nucleus mass is not spherical-symmetrically distributed, and there are additional forces such as gas drag, solar tides, and radiation pressure. We must understand to what extent our approximation affects the validity of our orbital element solutions.

Radiation pressure can be dismissed during the short timespan of the observations, since it is weaker by a factor $\sim 10^{5}$ than nucleus gravity for $0.1 \mathrm{~m}$ grains (e.g., Finson \& Probstein 1968). To test the effect of solar tides, and to a certain extent that of the gas drag, the position and velocity vectors of Rosetta 
itself at the beginning of the imaging sequence (according to the tabulated reconstructed orbit provided by ESOC) were used to calculate the orbital elements of the spacecraft. These hyperbolic ${ }^{2}$ elements were then used to predict the spacecraft position vector during the next $30 \mathrm{~min}$, only using the gravitational force of a nucleus point source, and comparing with the tabulated vectors. After $30 \mathrm{~min}$ and $0.62 \mathrm{~km}$ of travel, the discrepancy in nucleus distance was $0.17 \mathrm{~mm}$, and the true anomaly was off by $7.5 \times 10^{-4}$ degrees, translating into a $0.37 \mathrm{~m}$ position error. This may serve as an example of the penalty payed for not having considered solar tides and gas drag (at $28 \mathrm{~km}$ from the nucleus). Such a discrepancy, if suffered by a grain, would not be measurable since it corresponds to $\lesssim 0.3 \mathrm{px}$ at a distance of $\geq 10 \mathrm{~km}$.

However, the grains are located in different locations than Rosetta, where the gas drag is potentially stronger. Gas kinetic simulations of comet comae by Davidsson et al. (2010) using the direct simulation Monte Carlo (DSMC) technique, showed that the ratio $F_{\text {drag }} / F_{\text {grav }}$ between the gas drag force $F_{\text {drag }}$ on a particle moving much slower than the gas and the nucleus gravitational force $F_{\text {grav }}$ is constant above the gas acceleration zone (this zone is located within a few kilometers of the nucleus surface) and that it falls somewhat below this constant value within the acceleration zone. This applies for spherical gas expansion, which may be a reasonable assumption along a given radial direction within a few times $10 \mathrm{~km}$ of the nucleus, even when the nucleus is irregular and the outgassing rates vary across the surface. Thus, decreasing the distance between Rosetta and the nucleus would only have reduced the relative importance of gas drag even more. As long as nucleus outgassing in the direction of the grains is similar to that in the direction of Rosetta, differences in $r_{\text {com }}$ matter little.

However, it cannot be excluded that the outgassing of 67P has a strong directional dependence and that $F_{\text {drag }} / F_{\text {grav }}$ is higher in the direction of a grain than in the direction of Rosetta, as seen from the nucleus center. Analysis of September 2014 outgassing rates of $67 \mathrm{P}$ measured by Rosetta instruments is still ongoing at the time of writing, but if the early June $2014\left(r_{\mathrm{h}}=3.9 \mathrm{AU}\right)$ range of $5 \times 10^{24} \leq Q \leq 4 \times 10^{25}$ molec s$^{-1}$ (Gulkis 2014) is used as a guideline and this gas is assumed to emanate from an active surface area of 5\% (Davidsson \& Gutiérrez 2005) of that of a sphere with the same volume (Sierks et al. 2015) as 67P, a local production rate of $Z \sim 10^{19}$ molec $\mathrm{m}^{-2} \mathrm{~s}^{-1}$ is obtained. For such a $Z$-value, Davidsson et al. (2010) obtained $F_{\text {drag }} / F_{\text {grav }} \approx 0.1$ for compact bodies with negligible radial velocity. The dynamical effect on a grain that is subjected to an outward $F_{\text {drag }}$ that is $10 \%$ of the inward $F_{\text {grav }}$ is equivalent to reducing the nucleus mass to $90 \%$ of its actual value. To test the consequences of having a weaker effective nucleus gravity (caused by gas drag), the orbit element search was repeated for grain B, using a nucleus mass of $M_{\text {nuc }}=9 \times 10^{12} \mathrm{~kg}$. Since the kinetic energy of the grain essentially remains the same while the nucleus gravitational potential becomes less negative, this change is not expected to result in elliptic orbits for grain B. We find that the effect of nucleus mass reduction is to place the grain somewhat closer to the nucleus, adjust the semi-major axis and the eccentricity, but in such a manner that $q$ changes very little. The orientation of the orbit, the time of the pericenter passage, and the residuals are hardly affected. According to these two tests, our omission of gas drag or solar tides probably only weakly affects our osculating orbital solutions.

\footnotetext{
The orbit manoeuvre that for the first time placed Rosetta on a bound orbit at $r_{\text {com }}=29 \mathrm{~km}$ took place $4.5 \mathrm{~h}$ after the currently discussed OSIRIS observations were completed.
}

The nucleus of 67P is highly irregular (Sierks et al. 2015), and the considered $\propto r_{\text {com }}^{-1}$ gravitational potential may be a poor approximation close to the nucleus. However, we recall that the grains considered here travel short distances in their orbits during the $30 \mathrm{~min}$ of observation. For example, the change in the true anomaly $\Delta v$ amounts to $3.6^{\circ} \leq \Delta v \leq 11.5^{\circ}$ for the various grain B orbits. It is doubtful that the true gravity field diverges so strongly from the idealized $\propto r_{\text {com }}^{-1}$ potential during such short orbit segments that it would introduce severe errors in our orbital elements. Our conclusion is therefore that the $\propto r_{\text {com }}^{-1}$ gravitational potential is a good local approximation, meaning that our orbital elements are instantaneously valid and rather immune against modest changes in the modulus of the central force. We emphasize that they are osculating elements. They are valid around the epoch $T_{0}$, but there is no guarantee that a particle follows these trajectories long before or after $T_{0}$.

We thus continue the discussion under the pretext that the solutions are reliable. For grains A, C, and D, we derived bound elliptic orbits with pericenters well above the nucleus surface that might be explained by ejection followed by elevation of the pericenter by gas drag during the previous perihelion passage. This scenario is consistent with previous work (e.g., Richter \& Keller 1995; Fulle 1997; Scheeres \& Marzari 2000; Crifo et al. 2005; Molina et al. 2008). Alternatively, for grain D, we also find elliptic or hyperbolic orbits that intercept the nucleus surface. If these solutions remain valid when the trajectories are integrated back $\$ 3 \mathrm{~h}$ in time, they imply ejection from the nucleus surface. Forward integration will result in nucleus impact or escape, provided the orbital elements remain valid for at least $\sim 20 \mathrm{~h}$. We note that Fulle et al. (2010) predicted a maximum liftable grain diameter $d_{\max }=2.7-5.8 \mathrm{~mm}$ at a heliocentric distance $r_{\mathrm{h}}=3.4 \mathrm{AU}$, and $d_{\max }=0.12-0.27 \mathrm{~m}$ at $r_{\mathrm{h}}=3.2 \mathrm{AU}$, assuming a grain density of $\varrho=100 \mathrm{~kg} \mathrm{~m}^{-3}$. Fulle et al. (2010) did this by using coma, tail, and trail photometry to place empirical constraints on the highest ratio of radiation pressure to nucleus gravity force that is experienced by ejected grains. This force ratio can be converted into a maximum liftable grain diameter by assuming an escape velocity, which Fulle et al. (2010) took as $v_{\text {esc }}=0.5 \mathrm{~m} \mathrm{~s}^{-1}$. The strong change in $d_{\max }$ over a short $r_{\mathrm{h}}$ interval indicates that modest differences between actual local conditions on the nucleus surface in 2014 compared to previous apparitions, and compared to the nominal model assumptions of Fulle et al. (2010), may allow for the lifting of dm-sized grains even at $r_{\mathrm{h}}=3.39 \mathrm{AU}$. But ejection of dm-sized grains at 3.39 AU pre-perihelion cannot be considered a standard event according to the current understanding of dust ejection mechanisms. If it occurs, it requires special conditions, for example, a grain density substantially below the nucleus bulk value of $\varrho=470 \pm 45 \mathrm{~kg} \mathrm{~m}^{-3}$ (Sierks et al. 2015), and/or unusually strong local outgassing rates. We also note that the largest grains clearly identified by Rotundi et al. (2015) as escaping particles have diameters of at most $0.04 \mathrm{~m}$.

However, for grains A-C we also find hyperbolic orbits with pericenters well above the nucleus surface. Such solutions might have been disregarded as mathematically possible but physically unrealistic, had it not been for grain B - for this particle we find no other type of solution. There are at least two possible explanations for hyperbolic trajectories that do not intercept the nucleus surface:

1. The grain is a trail particle that experiences a low-velocity hyperbolic deflection by the nucleus. This means that the grain was lost from 67P a long time ago, it has traveled in a heliocentric orbit very similar to the orbit of the comet ever 
since, and suffered a close encounter with its parent body during OSIRIS observations. If so, the $T_{0}-T_{\text {per }}<0$ solutions that indicate that the grain had not yet reached its pericenter at the time of observation might be real.

2. If the trajectory is integrated back in time, using the orbit at epoch $T_{0}$ as initial conditions, but applying a more realistic force function that includes nucleus irregularity and gas drag, the orbit may change substantially on timescales $\gg 0.5 \mathrm{~h}$.

We first discuss option 1 . The probability that a dm-sized trail particle, with a velocity of $\sim 1 \mathrm{~m} \mathrm{~s}^{-1}$ relative to the comet, approaches the nucleus to within $30 \mathrm{~km}$ during the $30 \mathrm{~min}$ of observation, exceeds $1 \%$ if the number density of such grains is at least $2 \times 10^{-15} \mathrm{~m}^{-3}$. This is not a high probability, but sufficiently high to not be dismissed. This number density is three orders of magnitude lower than that of bound grains according to Rotundi et al. (2015). However, there is evidence indicating that the number density of large trail particles is even lower. According to Spitzer observations of the 67P dust trail analyzed by Kelley et al. (2009), the trail particles have a differential size distribution of $\propto d^{\gamma}$ with $\gamma=-3.5$ on the size interval $12 \mu \mathrm{m} \leq d \leq 1.2 \mathrm{~cm}$. Furthermore, if the flux observed by Spitzer was caused solely by mm-sized particles, their number density would have been $\sim 10^{-12} \mathrm{~m}^{-3}$ (Kelley et al. 2009). If $\gamma$ remains fixed when extending the interval to $\sim 0.1 \mathrm{~m}$, the number density of dm-sized particles consequently is $\sim 10^{-19} \mathrm{~m}^{-3}$. This is four orders of magnitude too low, meaning that the probability that OSIRIS observed a close encounter between the nucleus and a trail particle must be considered negligible. The probability is even lower if the $\gamma=-4.1$ slope obtained by Agarwal et al. (2007) is applied.

It is beyond the scope of this paper to explore option \#2. Such long-term orbit back-integrations may indicate that grain B (as well as the high- $q$ hyperbolic orbits of grains A and C) approach elliptic orbits in the hours or days before the OSIRIS observations. If this is the case, OSIRIS was potentially witnessing the onset of dispersal of the cloud of bound grains, which was a result of the increasing levels of activity as 67P approached the Sun. Alternatively, long-term orbit back-integrations may show that grain B originated from the nucleus surface in the recent past and that the nucleus-intercepting solutions for grains A and $\mathrm{D}$ remain valid up to pericenter as well. If this is the case, we may have to reconsider current thinking about the ejection of large dust grains far from the Sun.

\section{Conclusions}

The OSIRIS WAC on Rosetta observed four dm-sized grains in the inner coma of comet $67 \mathrm{P} /$ Churyumov-Gerasimenko on September 10, 2014. We determined osculating orbital elements with rather small residuals. Unfortunately, the observed arcs were too short to allow for unique solutions. For three of the grains, elliptic orbits were found that are consistent with the notion that a cloud of large bound grains builds up during perihelion and may survive for at least one heliocentric orbit. Hyperbolic escape trajectories can be found for all four grains, and this is the only known possibility for one grain. This may indicate that dispersal of large bound grains has been initiated already 3.39 AU pre-perihelion. It cannot be excluded that at least one grain has been ejected from the nucleus surface shortly before the OSIRIS observations, and if so, this has important implications for local outgassing conditions before strong waterdriven activity.
Acknowledgements. We are grateful to Herbert Raab for significant technical support on his software Astrometrica. OSIRIS was built by a consortium led by the Max-Planck-Institut für Sonnensystemforschung, Göttingen, Germany, in collaboration with CISAS, University of Padova, Italy, the Laboratoire d'Astrophysique de Marseille, France, the Instituto de Astrofísica de Andalucía, CSIC, Granada, Spain, the Scientific Support Office of the European Space Agency, Noordwijk, The Netherlands, the Instituto Nacional de Técnica Aeroespacial, Madrid, Spain, the Universidad Politéchnica de Madrid, Spain, the Department of Physics and Astronomy of Uppsala University, Sweden, and the Institut für Datentechnik und Kommunikationsnetze der Technischen Universität Braunschweig, Germany. The support of the national funding agencies of Germany (DLR), France (CNES), Italy (ASI), Spain (MEC), Sweden (SNSB), and the ESA Technical Directorate is gratefully acknowledged. We thank the Rosetta Science Ground Segment at ESAC, the Rosetta Mission Operations Centre at ESOC and the Rosetta Project at ESTEC for their outstanding work that enabled the science return of the Rosetta Mission.

\section{References}

Agarwal, J., Müller, M., Reach, W. T., et al. 2007, Icarus, 207, 992

A'Hearn, M. F., Belton, M. J. S., Delamere, W. A., et al. 2011, Science, 332, 1396

Bowell, E., Hapke, B., Domingue, D., et al. 1989, in Asteroids II, eds. R. P. Binzel, T. Gehrels, \& M. S. Matthews (Tucson: The University of Arizona Press), 524

Campbell, D. B., Harmon, J. K., \& Shapiro, I. I. 1989, ApJ, 338, 1094

Colina, L., Bohlin, R. C., \& Castelli, F. 1996, AJ, 112, 307

Crifo, J. F., Loukianov, G. A., Rodionov, A. V., \& Zakharov, V. V. 2005, Icarus, 176,192

Danby, J. M. A. 1989, Fundamentals of celestial mechanics (Richmond: Willmann-Bell, Inc.)

Davidsson, B. J. R., \& Gutiérrez, P. J. 2005, Icarus, 176, 453

Davidsson, B. J. R., Gulkis, S., Alexander, C., et al. 2010, Icarus, 210, 455

Finson, M. L., \& Probstein, R. F. 1968, ApJ, 154, 327

Fulle, M. 1997, A\&A, 325, 1237

Fulle, M., Colangeli, L., Agarwal, J., et al. 2010, A\&A, 522, A63

Goldstein, R. M., Jurgens, R. F., \& Sekanina, Z. 1984, AJ, 89, 1745

Gulkis, S. 2014, CBET, 3912, 1

Hermalyn, B., Farnham, T. L., Collins, S. M., et al. 2013, Icarus, 222, 625

Keller, H. U., Barbieri, C., Lamy, P., et al. 2007, Space Sci. Rev., 128, 433

Kelley, M. S., Wooden, D. H., Tubiana, C., et al. 2009, AJ, 137, 4633

Kelley, M. S., Lindler, D. J., Bodewits, D., et al. 2013, Icarus, 222, 634

Marsden, B. G. 1985, AJ, 90, 1541

Molina, A., Moreno, F., \& Jiménez-Fernaández, F. J. 2008, Earth Moon Planet, 102,521

Raab, H. 2012, Astrophysics Source Code Library [record ascl:1203.012]

Richter, K., \& Keller, H. U. 1995, Icarus, 114, 355

Richter, K., Curdt, W., \& Keller, H. U. 1991, A\&A, 250, 548

Rotundi, A., Sierks, H., Corte, V. D., et al. 2015, Science, 347, aaa3905

Sansaturio, M. E., Milani, A., \& Cattaneo, L. 1996, in Dynamics, ephemerides and astrometry of the solar system, eds. S. Ferraz-Mello, B. Morando, \& J.-E. Arlot, IAU Symp., 172, 193

Scheeres, D. J., \& Marzari, F. 2000, A\&A, 356, 747

Sierks, H., Barbieri, C., Lamy, P. L., et al. 2015, Science, 347, aaa1044

Smart, W. M. 1977, Textbook on spherical astronomy (Cambridge: Cambridge University Press)

Sykes, M. V., \& Walker, R. G. 1992, Icarus, 95, 180

Sykes, M. V., Lebofsky, L. A., Hunten, D. M., \& Low, F. 1986, Science, 232, 1115

Tubiana, C., Güttler, C., Kovacs, G., et al. 2015, A\&A, 583, A46

Turner, H. H. 1893, MNRAS, 54, 11

1 Department of Physics and Astronomy, Uppsala University, Box 516, 75120 Uppsala, Sweden e-mail: bjorn.davidsson@physics.uu.se

2 Instituto de Astrofísica de Andalucía (CSIC), c/ Glorieta de la Astronomía s/n, 18008 Granada, Spain

3 Max-Planck-Institut für Sonnensystemforschung, Justus-vonLiebig-Weg, 3, 37077 Göttingen, Germany

4 University of Padova, Department of Physics and Astronomy, Vicolo dell'Osservatorio 3, 35122 Padova, Italy

5 Laboratoire d'Astrophysique de Marseille, UMR 7326, CNRS \& Aix Marseille Université, 38 rue Frédéric Joliot-Curie, 13388 Marseille Cedex 13, France 
6 Centro de Astrobiologia, CSIC-INTA, 28850 Torrejon de Ardoz, Madrid, Spain

7 International Space Science Institute, Hallerstraße 6, 3012 Bern, Switzerland

8 Scientific Support Office, European Space Research and Technology Centre/ESA, Keplerlaan 1, Postbus 299, 2201 AZ Noordwijk ZH, The Netherlands

9 PAN Space Research Center, Bartycka 18A, 00716 Warszawa, Poland

10 Institut für Geophysik und extraterrestrische Physik (IGEP), Technische Universität Braunschweig, Mendelssohnstr. 3, 38106 Braunschweig, Germany

11 University of Maryland, Department of Astronomy, College Park, MD 20742-2421, USA

12 Gauss Professor, Akademie der Wissenschaften zu Göttingen and Max-Planck-Institut für Sonnensystemforschung, Justus-vonLiebig-Weg 3, 37077 Göttingen, Germany

13 LESIA-Observatoire de Paris, CNRS, Université Pierre et Marie Curie, Université Paris Diderot, 5 place J. Janssen, 92195 Meudon, France

14 LATMOS, CNRS/UVSQ/IPSL, 11 boulevard d'Alembert, 78280 Guyancourt, France

15 Centro di Ateneo di Studi ed Attivitá Spaziali "Giuseppe Colombo" (CISAS), University of Padova, via Venezia 15, 35131 Padova, Italy

16 INAF, Osservatorio Astronomico di Padova, Vicolo dell'Osservatorio 5, 35122 Padova, Italy

17 CNR-IFN UOS Padova LUXOR, via Trasea, 7, 35131 Padova, Italy
18 University of Trento, via Mesiano 77, 38100 Trento, Italy

19 Department of Industrial Engineering - University of Padova, via Venezia 1, 35131 Padova, Italy

20 INAF-Osservatorio Astronomico, via Tiepolo 11, 34014 Trieste, Italy

21 Aix Marseille Université, CNRS, LAM (Laboratoire d'Astrophysique de Marseille) UMR 7326, 13388 Marseille, France

22 Deutsches Zentrum für Luft- und Raumfahrt (DLR), Institut für Planetenforschung, Rutherfordstraße 2, 12489 Berlin, Germany

23 National Central University, Graduate Institute of Astronomy, 300 Chung-Da Rd, 32054 Chung-Li, Taiwan

24 Operations Department, European Space Astronomy Centre/ESA, PO Box 78, 28691 Villanueva de la Canada, Madrid, Spain

25 Centre for Astrophysics and Planetary Science, School of Physical Sciences, The University of Kent, Canterbury, CT2 7NH, UK

26 University of Padova, Department of Physics and Astronomy, via Marzolo 8, 35131 Padova, Italy

27 Institut für Datentechnik und Kommunikationsnetze der TU Braunschweig, Hans-Sommer-Str. 66, 38106 Braunschweig, Germany

28 University of Padova, Department of Information Engineering, via Gradenigo 6/B, 35131 Padova, Italy

29 Planetary and Space Sciences, Department of Physical Sciences, The Open University, Milton Keynes, MK7 6AA, UK

${ }^{30}$ Physikalisches Institut der Universität Bern, Sidlerstr. 5, 3012 Bern, Switzerland 